\title{
Multistage Nonlinear Blind Interference Cancellation for DS-CDMA Systems *
}

\author{
Dragan Samardzija \\ Bell Labs, Lucent Technologies, \\ 791 Holmdel-Keyport Road, \\ Holmdel, NJ 07733, USA \\ dragan@lucent.com
}

\author{
Narayan Mandayam, Ivan Seskar \\ WINLAB, Rutgers University, \\ 73 Brett Road, Piscataway NJ 08854, USA \\ narayan, seskar@winlab.rutgers.edu
}

\begin{abstract}
In this paper we propose a multistage nonlinear blind interference cancellation (MS-NL-BIC) receiver for direct-sequence code-division multiple-access (DS-CDMA) systems. The receiver uses higher order statistics of the received baseband signal. Specifically, we use the second and fourth moments of the received signal to determine a component of the received vector that has significant mean energy and low variability of the energy, both of which are favorable characteristics for application in an interference cancellation scheme that uses hard decisions. The structure of the receiver is multidimensional and can be viewed as a matrix of receivers. Each row in the matrix consists of receivers that perform (hard decisions) cancellation of successive components that have significant mean energy and low variability of the energy. The columns of the matrix essentially resemble multistage receivers that iteratively refine performance from earlier stages. Simulation results show that unlike linear receivers, the MS-NL-BIC is exceptionally efficient in systems with strong and highly correlated interferers, as may be the case in overloaded DS-CDMA systems.

Keywords: Blind interference cancellation, successive interference cancellation, iterative refinement.

\footnotetext{
${ }^{*}$ This work is supported in part by the New Jersey Commission on Science and Technology under the New
} Jersey Center for Wireless Communication Technologies. This paper was presented, in part, at VTC 2000, Boston, September 2000.
\end{abstract}




\section{Introduction}

One of the driving forces of the next generation of wireless communications is the demand for higher data rates and higher capacity of wireless systems. Primary applications of higher data rates seem to be in the downlink direction (for example, typical internet data traffic such as downloading of web pages). Furthermore, wideband direct-sequence code-division multiple-access (DS-CDMA) technology has emerged as one of the most promising candidates for future wireless systems (e.g., third generation systems $[1,2])$. It is therefore of great interest to investigate the performance of these systems and its viability for higher data rates envisioned in the future.

In DS-CDMA systems, in general, crosscorrelations between signature (spreading) sequences are nonzero. This results in the near-far effect where the multiple-access interference (MAI) can disrupt reception of a highly attenuated desired user signal [3]. Baseband signal processing techniques such as multiuser detection and interference cancellation have the potential to combat this problem and provide higher performance at the cost of increasing complexity of the receiver. Rapid progress in the area of semiconductor technology has resulted in a significant increase in the processing speeds of core technologies (DSP, FPGA and ASIC device). Advances in VLSI technology, and design of the algorithms that are optimized with respect to a specific implementation platform, are further narrowing the gap between the complexity of the algorithms and processing speeds (e.g., solutions that are presented in $[4,5])$. These and other developments suggest that the transceivers in future wireless systems will employ some form of interference mitigation.

Several multiuser receivers have been proposed (for example, see [6-9]). These receivers are denoted as centralized because they require knowledge of parameters (signature sequences, amplitudes and timing) for all users in the system. Therefore, they are more suitable for processing at the base station. For the downlink, it is desirable to devise decentralized receivers that exploit the knowledge of the desired user parameters only. The use of short signature sequences simplify the task of multiuser detection and interference cancellation, since a receiver can adaptively learn (estimate) the structure of the MAI [10]. Decentralized receivers may be further classified into data aided and nondata aided receivers. Data aided adaptive multiuser detection is an approach which does not require prior knowledge of the interference parameters. But, it requires a training data sequence for every active user. For example, adaptive receivers in $[7,11,12]$ are based on the MMSE criterion, 
and the one in [13] is based on minimizing probability of bit-error. More recently, decision feedback detectors using the MMSE criterion have been proposed [14,15]. Unlike data aided receivers, blind (or nondata aided) multiuser detectors require no training data sequence, but only knowledge of the desired user signature sequence and its timing. The receivers treat MAI and background noise as a random process, whose statistics must be estimated. Majority of blind multiuser detectors are based on estimation of second order statistics of the received signal. In [16], a blind adaptive MMSE multiuser detector is introduced (proven to be equivalent to the minimum output energy (MOE) detector). A subspace approach for blind multiuser detection is presented in [17]; where both the decorrelating and the MMSE detector are obtained blindly. Further, adaptive and blind solutions are analyzed in [18], with an overview in [10]. A blind successive interference cancellation (SIC) scheme, which uses second order statistics, is proposed in [19,20].

In this paper we propose a novel blind interference cancellation receiver, which assumes knowledge of only the desired user's signature sequence. The receiver is based on determining that component of the received signal that has significant mean energy and low variability in the energy. It applies the minimum variance of energy and maximum mean energy criterion (MVE-MME), which is described in Section 3. Furthermore, we analyze a relationship between the above criterion and Godard's dispersion function [21] and constant modulus (CM) criterion [22]. In Section 4, using the MVE-MME criterion, we derive a nonlinear multistage blind interference cancellation (NL-MS-BIC) receiver. The structure of the NL-MS-BIC receiver is multidimensional and can be viewed as a matrix of IC stages. Each row in the matrix consists of IC stages that perform the blind (hard decisions) successive interference cancellation. The columns of the matrix essentially resemble multistage receivers that iteratively refine performance from earlier stages. This particular multistage structure of the receiver allows concurrent (parallel) execution of the IC stages which makes it very suitable for implementation using multiprocessor DSP and/or FPGA (or ASIC) platform. Simulation results are presented in Section 5, and we conclude in Section 6. 


\section{Background}

The received baseband signal, $r(t)$, in a $K$-user asynchronous DS-CDMA additive white Gaussian noise (AWGN) system is

$$
r(t)=\sum_{i=-J}^{J} \sum_{k=1}^{K} A_{k} b_{k}(i) s_{k}\left(t-i T-\tau_{k}\right)+\sigma n(t)
$$

where $A_{k}$ is the received amplitude, $b_{k}(i) \in\{-1,+1\}$ is binary, independent and equiprobable data, $s_{k}(t)$ is the signature sequence which is assumed to have unit energy, $\tau_{k}$ is the relative time offset, all for the $k^{t h}$ user. $T$ is the symbol period and $n(t)$ is AWGN with unit power spectral density, with $\sigma$ being square root of the noise power. $2 J+1$ is the number of data symbols per user per frame.

It is well known that an asynchronous system with independent users can be analyzed as synchronous if equivalent synchronous users are introduced, which are effectively additional interferers [3]. In this paper we consider the received signal $r(t)$ over only one symbol period that is synchronous to the desired user $(k=1)$. The discrete representation for the received signal in $(1)$ can be written in vector form as

$$
\mathbf{r}=\sum_{k=1}^{L} A_{k} b_{k} \mathbf{s}_{k}+\sigma \mathbf{n}
$$

where the number of the interferers $(L-1=2(K-1))$ is doubled due to the equivalent synchronous user analysis. $\mathbf{r}, \mathbf{s}_{k}$ and $\mathbf{n}$ are vectors in $\Re^{M}$, where $M$ is the number of chips per bit.

Consider the nonlinear centralized SIC scheme which is presented in $[5,8]$. We now present a brief outline of the above scheme because its approach to nonlinear interference cancellation is generalized in this paper and later applied in a blind interference cancellation scheme (Section 4). In the nonlinear centralized SIC scheme $[5,8]$ it is assumed that the signature sequences are perfectly known (centralized approach). The basic operations of the SIC algorithm are (see Figure 1):

1. Detect one user with the conventional detector, i.e., matched filter (MF).

2. Regenerate the baseband signal (vector) for this user.

3. Cancel the regenerated signal (vector) from the received baseband signal. 
Then, this operation is repeated successively for all the users in the system. The idea is that successive cancellations result in reduced MAI for the remaining users. The received vector $\mathbf{r}_{j+1}$ after stage $j$ of the cancellation is given by

$$
\mathbf{r}_{j+1}=\mathbf{r}_{j}-\hat{A}_{j} \hat{b}_{j} \mathbf{s}_{j}
$$

where $\mathbf{r}_{j}$ is the received vector, $\hat{A}_{j}$ and $\hat{b}_{j}$ are the corresponding estimates of the amplitude and the bit, respectively, all for stage $j^{1}$. The above implementation of the SIC algorithm is nonlinear in that it uses hard decisions $\left(\hat{b}_{j} \in\{-1,1\}\right)$ in successive stages. A primary reason why the nonlinear centralized SIC cannot achieve performance of the single user lower bound (SULB) is due to an erroneous estimate of bit decision (i.e., $\hat{b}_{j} \neq b_{j}$ ). When an error happens, it causes the SIC scheme to double the interference, which is, of course, undesirable. Furthermore, the doubled interference propagates through the following IC stages, which degrades the overall performance of the receiver. For the same reason, this receiver is also not near-far resistant [3]. Further, imperfections in amplitude and delay estimates can lead to the non ideal regeneration and cancellation. Accordingly, to obtain best results, the user with the highest signal to interference ratio (SIR) should be cancelled first. This condition is usually relaxed and the user with highest received power is cancelled first, followed by the second strongest, and so forth $[8,9]$. Thus, it is desirable to identify users (or signature sequences) that have significant power (energy). Note that the SIC scheme requires amplitude estimates for the users, which implicitly requires low variability of the amplitude estimates for perfect cancellation.

Let us now generalize the nonlinear cancellation given by (3). In equation (3), let us replace $\mathbf{s}_{j}$ with a vector $\mathbf{v}_{j}$ (not necessarily a signature sequence). Furthermore, we replace $\hat{A}_{j}$ with the square root of the estimate of $\beta_{j}=E\left[\left(\mathbf{r}_{j}^{\top} \mathbf{v}_{j}\right)^{2}\right]$, and $\hat{b}_{j}$ with $\operatorname{sgn}\left(\mathbf{r}_{j}^{\top} \mathbf{v}_{j}\right)$. Thus, the nonlinear cancellation in the $j^{\text {th }}$ stage is executed as

$$
\mathbf{r}_{j+1}=\mathbf{r}_{j}-\sqrt{\hat{\beta}_{j}} \operatorname{sgn}\left(\mathbf{r}_{j}^{\top} \mathbf{v}_{j}\right) \mathbf{v}_{j}
$$

In the following, we propose favorable characteristics of the vector $\mathbf{v}_{j}$ to be successfully applied in the above scheme. We now analyze the estimate of the energy $\beta_{j}$ of the received signal in the

\footnotetext{
${ }^{1}$ Notation: $\hat{z}$ denotes an estimate of $z$
} 
direction of the vector $\mathbf{v}_{j}$. To estimate $\beta_{j}$ we use sample statistics as

$$
\hat{\beta}_{j}(n)=\frac{1}{N} \sum_{m=n-N+1}^{n}\left(\mathbf{r}^{\top}(m) \mathbf{v}_{j}\right)^{2}
$$

where $N$ is the size of the averaging window (number of samples), and $n$ and $m$ are time indices (will be omitted in the following). It is well known that the error of the estimate in (5) is directly related to the variance of $x_{j}=\left(\mathbf{r}^{\top} \mathbf{v}_{j}\right)^{2}$. Using the Chebyshev inequality [23] it can be shown that as the variance of $x_{j}$ gets lower, the accuracy (mean square error) of the energy estimate is improved:

$$
E\left[\left(x_{j}-E\left[x_{j}\right]\right)^{2}\right] \downarrow \Rightarrow E\left[\left(\hat{\beta}_{j}-\beta_{j}\right)^{2}\right] \downarrow
$$

We can say that for the vector $\mathbf{v}_{j}$ that corresponds to $x_{j}$ with lowest variance (among all vectors in $\Re^{M}$ ), the estimate of the energy is the most reliable, i.e., the mean square error of the energy estimate is the lowest. Note that the variance of $x_{j}$ is the variance of the energy of the received vector $\mathbf{r}$ in the direction of the vector $\mathbf{v}_{j}$ (i.e., variance of squared projection of the vector $\mathbf{r}$ onto the vector $\mathbf{v}_{j}$ ).

The above analysis leads us to believe that the vector $\mathbf{v}_{j}$ which corresponds to the low variability of the energy and significant mean energy of the vector $\mathbf{r}$ in the direction of $\mathbf{v}_{j}$, is desirable for the nonlinear cancellation given by (4). These characteristics of the vector $\mathbf{v}_{j}$ offer reliable estimates of the corresponding energy $\beta_{j}$ and sign of $\mathbf{r}^{\top} \mathbf{v}_{j}$. In the following we present a scheme that blindly determines (estimates) the vector $\mathbf{v}_{j}$ and further applies this vector to realize a multistage nonlinear interference cancellation scheme.

\section{MVE-MME Optimization Criterion}

We now present an optimization criterion which is used in deriving a nonlinear blind adaptive interference cancellation scheme. According to the analysis in Section 2, the goal of the optimization approach is to determine a component of the received vector $\mathbf{r}$ that has low variability in the energy and significant mean energy. We consider the squared output of the projection of $\mathbf{r}$ onto a vector 
$\mathbf{v} \in \Re^{M}$. The vector $\mathbf{v}$ is obtained from the following nonlinear procedure which is

$$
\mathbf{v}=\arg \min _{\mathbf{u}}\left\{\alpha(\mathbf{u})=(1-\mu) \alpha_{1}(\mathbf{u})-\mu \alpha_{2}(\mathbf{u})\right\}
$$

where $\mathbf{u} \in \Re^{M}$ is subject to $\mathbf{u}^{\top} \mathbf{u}=1$ and $0<\mu<1$. The function $\alpha_{1}(\mathbf{u})$ denotes the variance of the squared output $\mathbf{r}^{\top} \mathbf{u}$ and is given as

$$
\alpha_{1}(\mathbf{u})=E\left[\left(\left(\mathbf{r}^{\top} \mathbf{u}\right)^{2}-E\left[\left(\mathbf{r}^{\top} \mathbf{u}\right)^{2}\right]\right)^{2}\right]
$$

The function $\alpha_{2}(\mathbf{u})$ in (7) denotes the square mean energy given as

$$
\alpha_{2}(\mathbf{u})=\left(E\left[\left(\mathbf{r}^{\top} \mathbf{u}\right)^{2}\right]\right)^{2}
$$

Consider the function $\alpha_{1}(\mathbf{u})$. We now present the following proposition that gives an intuitive description of the minimum variance of energy criterion, which is obtained by minimizing the expression in (8).

Proposition 1 For the synchronous antipodal DS-CDMA system (described in (2)), with zero $A W G N(\sigma=0)$ and linearly independent signature sequences $\left\{\mathbf{s}_{i}\right\}_{i=1}^{L}$, the solutions for $\mathbf{w}=$ $\arg \min _{\mathbf{u}}\left(\alpha_{1}(\mathbf{u})\right)$, constrained as $\mathbf{u}^{\top} \mathbf{u}=1$, are classified in two groups:

(a) $\mathbf{w}= \pm \mathbf{d}_{i}(i=1, \cdots, L)$, where $\mathbf{d}_{i} \perp \operatorname{span}\left\{\mathbf{s}_{k}\right\}_{k=1, k \neq i}^{L}$ and $\mathbf{s}_{i}^{\top} \mathbf{d}_{i} \neq 0$. Actually, $\mathbf{d}_{i}$ is the modified matched filter that corresponds to the decorrelating detector for user $i$ [6].

(b) $\mathbf{w}$ is any vector from the noise subspace.

Further, the above solutions correspond to the absolute minimum of $\alpha_{1}(\mathbf{u})$ where it is zero.

We present a proof of the above proposition in the Appendix. Let us now compare $\alpha_{1}(\mathbf{u})$ with the following, well known, Godard's dispersion function [21]:

$$
J_{p}=\frac{1}{2 p} E\left[\left(\left(\mathbf{r}^{\top} \mathbf{u}\right)^{p}-\eta\right)^{2}\right]
$$

where $\eta$ is a real constant, and $p$ is an integer. For $\eta=E\left[\left(\mathbf{r}^{\top} \mathbf{u}\right)^{2}\right]$ and $p=2$, the cost function in (10) is directly proportional to $\alpha_{1}(\mathbf{u})$. In other words, $\alpha_{1}(\mathbf{u})$ penalizes dispersions of the squared output $\left(\mathbf{r}^{\top} \mathbf{u}\right)^{2}$ away from the constant $E\left[\left(\mathbf{r}^{\top} \mathbf{u}\right)^{2}\right]$. Furthermore, the well studied constant modulus (CM) 
cost function is defined as a special form of the function in equation (10), where $\eta=E\left[\left(\mathbf{r}^{\top} \mathbf{u}\right)^{4}\right] / \sigma^{2}$, and $p=2$. The CM cost function is widely used for blind equalization (see [22] and references therein). Later in this paper, $\alpha_{1}(\mathbf{u})$, which may be viewed as a slightly modified form of the CM cost function, is applied for blind interference cancellation in DS-CDMA systems. Let us now consider the function $\alpha_{2}(\mathbf{u})$. It can be shown that the vector $\mathbf{v}_{\max }=\arg \max _{\mathbf{u}}\left(\alpha_{2}(\mathbf{u})\right)$, constrained as $\mathbf{u}^{\top} \mathbf{u}=1$, is equal to the vector that also maximizes the mean energy $E\left[\left(\mathbf{r}^{\top} \mathbf{u}\right)^{2}\right]$. It is shown in $[19,20]$ that $\mathbf{v}_{\max }$ is the eigenvector that corresponds to the largest eigenvalue of the input covariance matrix $\mathbf{R}_{r}=E\left[\mathbf{r} \mathbf{r}^{\top}\right]$. Instead of the mean energy $E\left[\left(\mathbf{r}^{\top} \mathbf{u}\right)^{2}\right],\left(E\left[\left(\mathbf{r}^{\top} \mathbf{u}\right)^{2}\right]\right)^{2}$ is applied in (7) such that both terms $\left(\alpha_{1}()\right.$ and $\left.\alpha_{2}()\right)$ are of the same order (i.e., fourth order). Based on the above, the vector $\mathbf{v}$, which is defined in the equation (7), corresponds to that component of the received signal $\mathbf{r}$ that has low variability in the energy and significant mean energy. As discussed in Section 2, these characteristics are favorable for application of the vector $\mathbf{v}$ in a nonlinear interference cancellation scheme. The parameter $\mu$ is used to control which of these two characteristics (low variability of the energy or significant mean energy) is dominant. For example, if $\mu=0$, the optimization in (7) is equivalent to minimum variance of energy (MVE), and for $\mu=1$ it is equivalent to maximum mean energy (MME) optimization criterion. Therefore, we refer to (7) as the minimum variance of energy and maximum mean energy (MVE-MME) optimization criterion. Note that in Subsection 3.2 we revisit issues related to the parameter $\mu$ and propose its design.

\subsection{Adaptive Solution}

We now present an adaptive algorithm that solves (7). We exploit some properties of the functions given in (8) and (9). Let us assume that the input process $\mathbf{r}$ is wide sense stationary (WSS) and also that

$$
E\left[\left(\mathbf{r}^{\top}(n) \mathbf{u}\right)^{2}\left(\mathbf{r}^{\top}(n+m) \mathbf{u}\right)^{2}\right]=E\left[\left(\mathbf{r}^{\top}(n) \mathbf{u}\right)^{2}\right] E\left[\left(\mathbf{r}^{\top}(n+m) \mathbf{u}\right)^{2}\right]
$$

where $n$ and $m$ are time indices, and $n \neq m$. In other words, we assume that the energy of $\mathbf{r}$ in direction of the vector $\mathbf{u}$ is uncorrelated in different symbol (bit) intervals. Using the properties of 
WSS processes and (11) we can show that (8) can be written as

$$
\alpha_{1}(\mathbf{u})=\frac{1}{2} E\left[\left(\left(\mathbf{r}^{\top}(n) \mathbf{u}\right)^{2}-\left(\mathbf{r}^{\top}(n+m) \mathbf{u}\right)^{2}\right)^{2}\right]
$$

for all integer $n$ and $m, n \neq m$. Similarly, the expression (9) can be written as

$$
\alpha_{2}(\mathbf{u})=E\left[\left(\mathbf{r}^{\top}(n) \mathbf{u}\right)^{2}\right] E\left[\left(\mathbf{r}^{\top}(n+m) \mathbf{u}\right)^{2}\right]
$$

According to (12) and (13), and using sample statistics, the function $f(\mathbf{u})$ is defined as an approximation of $\alpha(\mathbf{u})$ as

$$
f(\mathbf{u}, n)=\frac{1}{F} \sum_{m=1}^{F}\left[\frac{1}{2}(1-\mu)\left(\left(\mathbf{r}^{\top}(n) \mathbf{u}\right)^{2}-\left(\mathbf{r}^{\top}(n+m) \mathbf{u}\right)^{2}\right)^{2}-\mu\left(\mathbf{r}^{\top}(n) \mathbf{u}\right)^{2}\left(\mathbf{r}^{\top}(n+m) \mathbf{u}\right)^{2}\right]
$$

where $F$ is the number of consecutive symbols used for the approximation. Gradient of $f(\mathbf{u}, n)$ is defined as

$$
\begin{aligned}
\nabla(f(\mathbf{u}, n)) & =\frac{2}{F} \sum_{m=1}^{F}\left\{(1-\mu)\left[\left(\mathbf{r}^{\top}(n) \mathbf{u}\right)^{3} \mathbf{r}(n)+\left(\mathbf{r}^{\top}(n+m) \mathbf{u}\right)^{3} \mathbf{r}(n+m)\right]-\right. \\
& \left.-\left[\left(\mathbf{r}^{\top}(n) \mathbf{u}\right)\left(\mathbf{r}^{\top}(n+m) \mathbf{u}\right)^{2} \mathbf{r}(n)+\left(\mathbf{r}^{\top}(n) \mathbf{u}\right)^{2}\left(\mathbf{r}^{\top}(n+m) \mathbf{u}\right) \mathbf{r}(n+m)\right]\right\}
\end{aligned}
$$

We can use a stochastic gradient algorithm [24] that solves (7) as

$$
\hat{\mathbf{v}}^{l+1}=\hat{\mathbf{v}}^{l}-\gamma \nabla\left(f\left(\hat{\mathbf{v}}^{l}, l\right)\right)
$$

where $l$ is the index of the iteration step, and $0<\gamma<1$ is a certain scalar which defines the length of the adaptation step. The constraint $\left|\hat{\mathbf{v}}^{l+1}\right|=1$ is forced after every iteration, where $\hat{\mathbf{v}}^{l}$ stands for the estimate of $\mathbf{v}$ in $l^{\text {th }}$ iteration step. 


\subsection{Choice of Parameter $\mu$}

As addressed earlier, the parameter $\mu$ is used to control which of the two characteristics of $\mathbf{v}$ (low variability of the energy or significant mean energy) is dominant. We choose $\mu$ as

$$
\mu=\mu(\mathbf{u})=\frac{\left(E\left[\left(\mathbf{r}^{\top} \mathbf{u}\right)^{2}\right]\right)^{2}}{E\left[\left(\mathbf{r}^{\top} \mathbf{u}\right)^{4}\right]}
$$

Note that the above definition is similar to the inverse of the normalized kurtosis [23] defined as $k_{s}=E\left[\left(\mathbf{r}^{\top} \mathbf{u}\right)^{4}\right] / \sigma^{4}$, but further analysis of this relationship is beyond the scope of this paper. Furthermore, as an approximation of the above definition, we set

$$
\mu(\mathbf{u}, n)=\frac{\sum_{m=1}^{F}\left(\mathbf{r}^{\top}(n) \mathbf{u}\right)^{2}\left(\mathbf{r}^{\top}(n+m) \mathbf{u}\right)^{2}}{\sum_{m=1}^{F}\left[\left(\mathbf{r}^{\top}(n) \mathbf{u}\right)^{4}+\left(\mathbf{r}^{\top}(n+m) \mathbf{u}\right)^{4}\right] / 2}
$$

in equation (15). Considering characteristics of the parameter $\mu$ that is defined by (17), it can be shown that

1. If $\mathbf{r}^{\top} \mathbf{u}$ is a real-valued Gaussian random process, $\mu$ is $1 / 3$.

2. Let $\mu_{M}$ denote $\mu$ corresponding to $\mathbf{r}^{\top} \mathbf{u}$, which is a uniform discrete real-valued M-ary random process, i.e., $\mathbf{r}^{\top} \mathbf{u} \in\left\{a_{i} \mid a_{i}=A(-1+2(i-1) /(M-1)), i=1, \cdots, M\right\}$, where $A$ is the maximum absolute value of $\mathbf{r}^{\top} \mathbf{u}$. Based on the above definition, it can be shown that

$$
\mu_{M}=\frac{1}{M} \frac{\left(\sum_{i=1}^{M} a_{i}^{2}\right)^{2}}{\sum_{i=1}^{M} a_{i}^{4}}
$$

Figure 2 depicts the parameter $\mu$ as a function of the alphabet size of a uniform, real-valued Mary random process. As a reference, we present $\mu$ that corresponds to a continuous uniformly distributed random process (denoted as CU), and a Gaussian random process (denoted as GP). Note that the function is decreasing with alphabet size $M$, or in other words,

$$
\mu_{M}>\mu_{M+1}
$$

Furthermore, we may note that $\mu$ is maximum at $M=2\left(\mu_{2}=1\right)$, i.e., for a real-valued bipolar random process. In addition, we note $\mu_{M}$ in equation (19) converges towards $\mu$ that 
corresponds to the continuous uniformly distributed random process ( $\mathrm{CU}$ in Figure 2).

From the above properties of the parameter $\mu$, we may draw the following conclusions. When the received signal at the output of the correlator, $\mathbf{r}^{\top} \mathbf{u}$, is a real-valued Gaussian random process (i.e., $\mathbf{u}$ lies in the noise subspace of the received vector $\mathbf{r}$ ), then $\mu$ takes a value close to its minimum thereby steering the MVE-MME criterion towards minimizing variance of energy (MVE). On the other hand, when the output $\mathbf{r}^{\top} \mathbf{u}$ is a close to a discrete-valued random process (as in the case when MAI dominates), $\mu$ approaches its maximum value thus steering the MVE-MME criterion towards maximizing mean energy (MME). In the course of adaptation, the value of $\mu$ given in equation (18) changes according to the projection $\mathbf{r}^{\top} \mathbf{u}$, i.e., $\mathbf{u}$ being in the noise (Gaussian) part of the signal subspace or the interference (discrete-valued random process) subspace.

\section{Application of the MVE-MME Criterion in the Multi- stage Nonlinear Blind IC Receiver}

We now present a multistage nonlinear blind interference canceler, denoted as MS-NL-BIC. The structure of the receiver is multidimensional and can be viewed as a matrix of receivers (i.e., matrix of IC stages). The MS-NL-BIC receiver consists of $P$ rows and $Q$ columns, where each entry of the matrix corresponds to an interference cancellation stage denoted as $\mathbf{I C}_{i j}(i=1, \ldots, P, j=1, \ldots, Q)$. The following steps are executed in the $\mathbf{I C}_{i j}$ stage (where $\mathbf{r}_{i j}$ is the input vector to that stage):

1. Add back $\mathbf{x}_{(i-1) j}$ as

$$
\mathbf{r}_{i j}^{\prime}=\mathbf{r}_{i j}+\mathbf{x}_{(i-1) j}
$$

where $\mathbf{x}_{(i-1) j}$ is a portion of the received signal that is cancelled in the $\mathbf{I C}_{i-1 j}$ stage. Note that the $\mathbf{I C}_{i-1 j}$ stage is the same column, but earlier row of the matrix. For the first row $(i=1)$, $\mathbf{x}_{0 j}=0(j=1, \cdots, Q)$ and $\mathbf{r}_{11}=\mathbf{r}$, because no cancellation is performed prior to this row.

2. Use adaptation rule in (16) $\left(\mathbf{r}_{i j}^{\prime}\right.$ replaces $\mathbf{r}$ ) to estimate $\mathbf{v}_{i j}$ as $\hat{\mathbf{v}}_{i j}$ (see Figure 3 ). Note that the vector $\hat{\mathbf{v}}_{i j}$ is further processed in the very same manner as an interferer signature sequence in the case of the nonlinear centralized SIC scheme (see Section 2). 
3. Estimate the energy $\beta_{i j}=E\left[\left(\mathbf{r}_{i j}^{\prime}{ }^{\top} \hat{\mathbf{v}}_{i j}\right)^{2}\right]$. Note that the estimation should be reliable because $\hat{\mathbf{v}}_{i j}$, as a component of the vector $\mathbf{r}_{i j}^{\prime}$, has low variability in the energy (due to the term $(1-\mu) \alpha_{1}(\mathbf{u})$ in $\left.(7)\right)$.

4. Detect the sign of $\mathbf{r}_{i j}^{\prime}{ }^{\top} \hat{\mathbf{v}}_{i j}$. Note that detection should be reliable, because the component $\hat{\mathbf{v}}_{i j}$ has significant mean energy (due to the term $-\mu \alpha_{2}(\mathbf{u})$ in $(7)$ ) and low variability.

5. Perform nonlinear cancellation as

$$
\mathbf{r}_{i j+1}=\mathbf{r}_{i j}^{\prime}-\mathbf{x}_{i j}
$$

where (see Figure 4)

$$
\mathbf{x}_{i j}=\operatorname{sgn}\left(\mathbf{r}_{i j}^{\prime \top} \hat{\mathbf{v}}_{i j}\right) \sqrt{\hat{\beta}_{i j}} \hat{\mathbf{v}}_{i j}
$$

The above procedure is executed successively (within the $i^{\text {th }}$ row of the matrix), where for the new stage $\mathbf{I C}_{i j+1}$, the input vector is $\mathbf{r}_{i j+1}$ (see equation (22)). The structure of the $i^{\text {th }}$ row (i.e., horizontal topology) is depicted in Figure 5. From the above, each row may be viewed as a blind equivalent to the nonlinear centralized SIC scheme, where the components $\hat{\mathbf{v}}_{i j}$ replace the actual signature sequence. After a sufficient number $Q$ of the stages in the $i^{\text {th }}$ row, cancellation is repeated in the $(i+1)^{t h}$ row (see Figure 6 ). The input vector $\mathbf{r}_{i+11}$ of the $(i+1)^{t h}$ row is $\mathbf{r}_{i Q+1}$. The stage $\mathbf{I} \mathbf{C}_{i+1 j}$ is used to iteratively refine the cancellation which is executed in the earlier stage $\mathbf{I} \mathbf{C}_{i j}$ $(j=1, \ldots, Q)$. With appropriate delay, the vector $\mathbf{x}_{i j}$, that is canceled in the stage $\mathbf{I} \mathbf{C}_{i j}$ is added back (step 1), and within the stage $\mathbf{I C}_{i+1 j}$ processing is performed again (steps 2 to 5 ).

In Section 5, $Q$ is selected to be equal to the number of dominant interferers, but in the more general case, this number might not be known at the receiver. A number of different schemes can be employed in order to determine the number of IC stages within each row of this receiver. Here, we propose the following simple scheme. In the first row $i=1$, the stage $\mathbf{I C}_{1 j}$ may be determined as the last stage in the row $(Q=j)$, if the estimate of the energy $\hat{\beta}_{1 j}$ drops below a certain threshold $T_{e}$. In other words

$$
\hat{\beta}_{1 j}<T_{e} \Rightarrow Q=j
$$

This simple scheme assumes that $\hat{\beta}_{i j} \geq \hat{\beta}_{i j+1}$ (i.e., the energy estimate is decreasing with column index $j$ ). In addition, the scheme is based on the assumption that the component $\hat{\mathbf{v}}_{i j}$, that corresponds to the mean energy $\beta_{i j}=E\left[\left(\mathbf{r}_{i j}^{\prime}{ }^{\top} \hat{\mathbf{v}}_{i j}\right)^{2}\right]$, which is below the threshold $T_{e}$, is not relevant for 
the cancellation. Furthermore, the number of the rows $P$ is directly related to the performance of the receiver. Thus, the trade-off in performance versus complexity can be controlled by the number $P$. After a sufficient number $P$ of the rows, detection of the desired user is performed using a linear detector (e.g., matched filter).

Note that implicit in the above algorithm is the assumption that the interferers are stronger than the desired user. If the desired user is strong, then additional processing is required to ensure that the desired signal is not canceled out before the detection. Briefly, we propose a corresponding scheme which is based on a threshold rule. In the last row, $i=P$, each vector $\hat{\mathbf{v}}_{P j},(j=1, \cdots Q)$ is projected onto the desired user signature sequence $\mathbf{s}_{1}$. The absolute value of the projection is compared against a predefined threshold $T_{h}\left(0<T_{h}<1\right)$. If the absolute value exceeds the threshold $T_{h}$,

$$
\left|\hat{\mathbf{v}}_{P j}^{\top} \mathbf{s}_{1}\right|>T_{h}
$$

the vector $\mathbf{x}_{P j}$ (defined in equation $(23)$, where $i=P$ ) should be added back to $\mathbf{r}_{P Q+1}$. Having inspected all the vectors $\hat{\mathbf{v}}_{P j},(j=1, \cdots Q)$, the addition is performed as follows

$$
\mathbf{r}_{P Q+1}^{\prime}=\mathbf{r}_{P Q+1}+\sum_{j \in E} \mathbf{x}_{P j}
$$

where the $j \in E$ corresponds to all vectors $\hat{\mathbf{v}}_{P j}$ that have met the criterion in (25). Further, the linear detection of the desired user is performed using $\mathbf{r}_{P Q+1}^{\prime}$ as input signal.

Note that a centralized multiuser detection scheme, which is proposed in [2,25], applies a similar iterative (recursive) refinement approach that is presented above. That particular scheme executes centralized SIC and iterative refinement in order to improve channel estimates for the users in the system. Unlike the MS-NL-BIC receiver, the scheme in [2,25] assumes the knowledge of all signature sequences of the users in the system (i.e., it is not blind). Further, the multistage structure of the receiver allows concurrent (parallel) execution of the IC stages. This inherent parallelism of the algorithm is a favorable characteristic for its implementation using multiprocessor DSP and/or FPGA (or ASIC) platform (see [4,5] and references therein). 


\section{Simulation Results}

We consider a synchronous AWGN DS-CDMA system using randomly generated signature sequences with processing gain $M=8$. The users are independent and the following cases are analyzed:

1. System with $L=8$ users (fully loaded), and equal-energy interferers: $A_{i}^{2} / A_{1}^{2}=25, i=2, \cdots, 8$.

2. System with $L=4$ users, and equal-energy interferers: $A_{i}^{2} / A_{1}^{2}=25, i=2, \cdots, 4$.

3. System with $L=12$ users (overloaded system); three strong equal-energy interferers: $A_{i}^{2} / A_{1}^{2}=$ $25, i=2, \cdots, 4$, and eight interferers with the same energy as the desired user: $A_{i}^{2} / A_{1}^{2}=1, i=$ $5, \cdots, 12$.

The crosscorrelation profile of the users with respect to the desired user is depicted in Figure 7. Note that in this particular example the crosscorrelations are very high, except for users 5,10 and 12 which happen to be orthogonal to the desired user. In the case 1, the system has users $i=1, \cdots, 8$, and in the case 2 only $i=1, \cdots, 4$. In all our results the input sample covariance matrix is estimated according to

$$
\hat{\mathbf{R}}_{r}(i)=\frac{1}{N} \sum_{k=i-N+1}^{i} \mathbf{r}(k) \mathbf{r}^{\top}(k)
$$

where $N$ is the size of the averaging window (number of samples), and $i$ is the time index (will be omitted in the following text). Performance of the conventional matched filter (MF), the centralized MMSE receiver (denoted as MMSE), the blind MMSE receiver (BMMSE) (detector $\hat{\mathbf{c}}=\hat{\mathbf{R}}_{r}^{-1} \mathbf{s}_{\mathbf{1}}[17]$ ) and the single user lower bound (SULB) are used as benchmarks for evaluation of the MS-NL$\mathrm{BIC}$ receiver. The centralized MMSE assumes perfect knowledge of all the signature sequences, amplitudes and the variance of the AWGN. Performance of the MS-NL-BIC is evaluated for MF (MS-NL-BIC-(MF)) and the blind MMSE (MS-NL-BIC-(MMSE)); where these linear detectors are used for detection of the desired user after the cancellation (after $P$ rows and $Q$ IC stages within each row). The MS-NL-BIC-(MMSE) uses the sample covariance matrix of the output signal of the last IC stage $\left(\mathbf{I C}_{P Q}\right)$. Note that the desired user energy is set to be much lower than the energy of the interferers, and, as discussed in Section 4, the prevention of the excessive cancellation of the desired user is not performed. In each IC stage, the performance is measured after 1000 symbols 
used for the estimation in (16) and (27), and $F=5$ in (14), (15) and (18). Regarding the parameter $\mu$, we apply the approximation given by (18). We assume the knowledge of the number of dominant interferers $Q$, which is the number of columns of the receiver matrix.

For the case 1, Figure 8.a depicts bit-error rate (BER) as a function of signal to background noise ratio (SNR) (with respect to the desired user). The results are obtained after a total of $P=4$ rows and $Q=7$ columns, which is where the BER reaches minimum. Additional IC stages do not introduce any improvement for this particular example. For $S N R=8 d B$, BER versus total number of IC stages is presented in Figure 8.b. Note that in this example the MS-NL-BIC(MMSE) performs better than the MS-NL-BIC(MF). In this case, after the last IC stage $\left(\mathbf{I C}_{47}\right)$ MAI is still present, therefore, BMMSE detector can further improve the performance of the MS-NL-BIC receiver in the case 1 .

Equivalent results, for the case 2, with $P=4$ and $Q=3$ and $S N R=8 d B$ are shown in Figures 9.a and 9.b, respectively. In Figure 9.b, note that the MS-NL-BIC(MMSE) converges faster with respect to number of IC stages, but, at the end the MS-NL-BIC(MF) offers lower BER for this particular example. In this case, after the last IC stage $\left(\mathbf{I C}_{43}\right)$, MAI is almost completely removed. Introduction of the BMMSE as linear detector in the MS-NL-BIC receiver, may cause a drop in the performance due to estimation errors of the covariance matrix (in (27)) which is used to derive the BMMSE detector (this particular topic is analyzed in [20]).

We consider the performance of our receiver in the case 3, which is an overloaded DS-CDMA system. Figure 10 depicts BER versus SNR (with respect to the desired user). The same figure presents the performance of the matched filter (denoted as MF-8) for the system without the strong interferers (only the desired user and eight equal-energy interferers, which is identical to ideal cancellation of the users 2, 3 and 4). The receiver with $Q=3$ and $P=4$ is used. From the results in Figure 10, we note that the MS-NL-BIC completely cancels the strong users i.e, the MSNL-BIC(MF) performance is identical to the MF-8 performance (in Figure 10 their characteristics overlap).

From these results, it is seen that the MS-NL-BIC outperforms linear receivers (MF and BMMSE), significantly. The performance of the linear receivers is expected because it is well known that they do not perform well in the case of the systems with strong and highly correlated interferers (with respect to the desired user signature sequence) [3], as may be the case in overloaded systems. 
But, these results suggest that the MS-NL-BIC may be applied as a blind solution in the case of overloaded systems with strong interferers.

Let us now study the characteristics of the estimates $\hat{\mathbf{v}}_{i j}(i=1, \ldots, P, j=1, \ldots, Q)$. In all cases that we have observed, as the processing progresses from row to row, estimates $\hat{\mathbf{v}}_{i j}$ within each column of the receiver matrix, approach one of the actual signature sequences (with sign ambiguity). Each column corresponds to a different signature sequence. For example, in the case 1, we observe how the estimates $\hat{\mathbf{v}}_{i j}$ within columns $j=1,4,7$ approach the actual signature sequences $l=2,5,4$, respectively. The absolute value of the crosscorrelation $\hat{\mathbf{v}}_{i j}^{\top} \mathbf{s}_{l}$ is depicted in Figure 11, where the abscissa represents the index of the row $(i=1, \cdots, 4)$. The results appear to be similar for all columns $j=1, \cdots, 7$. Note that in the last row, the estimates are practically identical to the signature sequences i.e., the absolute value of the normalized crosscorrelation is 1 . We have consistently observed these results in our simulations, and a detailed mathematical analysis and explanation of the above phenomenon is of future interest.

\section{Conclusion}

We have introduced the MVE-MME optimization criterion which is then used to implement the MSNL-BIC receiver. The receiver is based on determining the component of the received vector that has significant mean energy and low variability in the energy. The MS-NL-BIC consists of multiple IC stages, and can be viewed as a matrix of IC stages. The columns of the matrix resemble multistage receivers that iteratively refine performance from earlier stages, while each row corresponds to a blind equivalent of the nonlinear centralized SIC scheme. The ability of the receiver to exceed the performance of linear receivers is observed via simulation results. It is seen that this scheme is particularly effective for systems with very strong interferers which are strongly correlated with the desired user signature sequence. Therefore, this may be a very viable solution for implementation in the case of overloaded systems with strong interferers. 


\section{Appendix: Proof of Proposition 1}

Consider a synchronous antipodal DS-CDMA system, with zero AWGN $(\sigma=0)$ and linearly independent signature sequences. Let us denote a component of the signature sequence $\mathbf{s}_{i}$ as $\mathbf{d}_{i}\left(\mathbf{d}_{i}^{\top} \mathbf{d}_{i}=\right.$ 1), which is orthogonal to other users' signature sequences, or in other words, $\mathbf{d}_{i} \perp \operatorname{span}\left\{\mathbf{s}_{k}\right\}_{k=1, k \neq i}^{L}$ and $\mathbf{s}_{i}^{\top} \mathbf{d}_{i} \neq 0$, which can be written as

$$
\mathbf{s}_{j}^{\top} \mathbf{d}_{i}=c_{i} \delta_{i j}, i, j=1, \cdots, L
$$

where $\left|c_{i}\right| \leq 1$ and $c_{i} \neq 0$, and $\delta_{i j}$ is the Kronecker delta function. Projection of the received vector $\mathbf{r}$ (see equation (2)) on $\mathbf{d}_{i}$ yields (in the absence of additive background noise):

$$
\mathbf{r}^{\top} \mathbf{d}_{i}=A_{i} b_{i} c_{i}
$$

where $A_{i}$ and $b_{i}$ are the amplitude and bit, respectively, all for user $i$. From (29), it follows that $\left(\mathbf{r}^{\top} \mathbf{d}_{i}\right)^{2}=E\left[\left(\mathbf{r}^{\top} \mathbf{d}_{i}\right)^{2}\right]=$ const, which results in

$$
E\left[\left(\left(\mathbf{r}^{\top} \mathbf{d}_{i}\right)^{2}-E\left[\left(\mathbf{r}^{\top} \mathbf{d}_{i}\right)^{2}\right]\right)^{2}\right]=0
$$

Note that

$$
\alpha_{1}(\mathbf{u})=E\left[\left(\left(\mathbf{r}^{\top} \mathbf{u}\right)^{2}-E\left[\left(\mathbf{r}^{\top} \mathbf{u}\right)^{2}\right]\right)^{2}\right] \geq 0, \forall \mathbf{u}
$$

Therefore, the absolute minimum of $\alpha_{1}(\mathbf{u})$ is zero. Based on (30) and (31), $\alpha_{1}(\mathbf{u})$ reaches the absolute minimum for $\mathbf{d}_{i}(i=1, \cdots, L)$. Using the above approach, the same result can be obtained for $-\mathbf{d}_{i}(i=1, \cdots, L)$. This proves part (a) of Proposition 1.

Further, if $\mathbf{N}$ denotes the noise subspace and $\sigma=0$, then by definition of the noise subspace [26], it follows that

$$
\mathbf{r}^{\top} \mathbf{u}=0, \forall \mathbf{u} \in \mathbf{N}, \mathbf{u}^{\top} \mathbf{u}=1
$$

Consequently

$$
\alpha_{1}(\mathbf{u})=0, \forall \mathbf{u} \in \mathbf{N}, \quad \mathbf{u}^{\top} \mathbf{u}=1
$$

which proves that for any vector $\mathbf{u}$ from the noise subspace, $\alpha_{1}(\mathbf{u})$ reaches the absolute minimum 
of zero. This concludes proof for part (b) of Proposition 1. 


\section{References}

[1] T. Ojanpera and R. Prasad, "An Overview of Air Interface Multiple Access for IMT2000/UMTS," IEEE Communication Magazine, vol. 36, pp. 82 - 95, September 1998.

[2] F. Adachi, M. Sawahashi, and H. Suda, "Wideband DS-CDMA for Next-Generation Mobile Communications Systems," IEEE Communication Magazine, vol. 36, pp. 56 - 69, September 1998.

[3] S. Verdú, Multiuser Detection. Cambridge University Press, 1998.

[4] I. Seskar and N. Mandayam, "Software-Defined Radio Architectures for Interference Cancellation in DS-CDMA Systems," IEEE Personal Communications, vol. 6, no. 4, pp. 26-34, 1999.

[5] I. Seskar, K. Pedersen, T. Kolding, and J. Holtzman, "Implementation Aspects for Successive Interference Cancellation in DS/CDMA Systems," Wireless Networks, no. 4, pp. 447-452, 1998.

[6] R. Lupas and S. Verdú, "Linear Multiuser Detectors for Synchronous Code-Division MultipleAccess Channels," IEEE Transactions on Information Theory, vol. 35, pp. 123-136, January 1989.

[7] U. Madhow and M. Honig, "MMSE Interference Suppression for Direct-Sequence SpreadSpectrum CDMA," IEEE Transactions on Communications, vol. 42, pp. 3178-3188, December 1994.

[8] P. Patel and J. Holtzman, "Analysis of a Simple Successive Interference Cancellation Scheme in DS/CDMA Systems," IEEE JSAC, Special Issue on CDMA, vol. 12, pp. 796-807, June 1994.

[9] A. Duel-Hallen, "A Family of Multiuser Decision-Feedback Detectors for Asynchronous CodeDivision Multiple-Access Channels," IEEE Transactions on Communications, vol. 43, pp. 421434, Feb./March/April 1995.

[10] U. Madhow, "Blind Adaptive Interference Suppression for Direct-Sequence CDMA," IEEE Proceedings, Special Issue on Blind Identification and Equalization, pp. 2049-2069, October 1998. 
[11] S. Miller, "An Adaptive Direct-Sequence Code-Division Multiple-Access Receiver for Multiuser Interference Rejection," IEEE Transactions on Communications, vol. 43, pp. 1746-1755, February/March/April 1995.

[12] P. Rapajic and B. Vucetic, "Adaptive Receiver Structure for Asynchronous CDMA Systems," IEEE JSAC, vol. 12, pp. 685 - 697, May 1994.

[13] N. Mandayam and B. Aazhang, "Gradient Estimation for Sensitivity Analysis and Adaptive Multiuser Interference Rejection in Code Division Multiple Access Systems," IEEE Transactions on Communications, vol. 45, pp. 848-858, July 1997.

[14] G. Woodward, R. Ratasuk, and M. Honig, "Multistage Multiuser Decision Feedback Detection for DS-CDMA," International Conference on Communications, vol. 1, pp. 68-72, June 1999. Vancouver.

[15] R. Ratasuk, G. Woodward, and M. Honig, "Adaptive Multiuser Decision Feedback for Asynchronous Cellular DS-CDMA," 37th Allerton Conference, 1999.

[16] M. Honig, U. Madhow, and S. Verdú, "Blind Adaptive Multiuser Detection," IEEE Transactions on Information Theory, vol. 41, pp. 944-960, July 1995.

[17] X. Wang and V. Poor, "Blind Multiuser Detection: A Subspace Approach," IEEE Transactions on Information Theory, vol. 44, pp. 677-690, March 1998.

[18] S. Ulukus and R. Yates, "A Blind Adaptive Decorrelating Detector for CDMA Systems," IEEE JSAC, vol. 16, pp. 1530-1541, October 1998.

[19] D. Samardzija, N. Mandayam, and I. Seskar, "Blind Interference Cancellation for the Downlink of CDMA Systems," Conference on Information Sciences and Systems, vol. 2, pp. TP3.17TP3.22, March 2000. Princeton University.

[20] D. Samardzija, N. Mandayam, and I. Seskar, "Blind Successive Interference Cancellation for DS-CDMA Systems," To Appear in IEEE Transactions on Communications, 2001. 
[21] D. Godard, "Self-Recovering Equalization and Carrier Tracking in Two-Dimensional Communication Systems," IEEE Transactions on Communications, vol. 28, no. 11, pp. 1867-1875, 1980.

[22] S. Haykin, ed., Unsupervised Adaptive Filtering, Blind Deconvolution, vol. 2. Wiley Interscience, first ed., 2000.

[23] B. Picinbono, Random Signals and Systems. Prentice Hall, 1993.

[24] Y. Z. Tsypkin, Adaptation and Learning in Automatic Systems. Academic Press, 1971.

[25] M. Sawahashi and et al., "Pilot Symbol-Aided Coherent Multistage Interference Canceller Using Recursive Channel Estimation for DS-CDMA Mobile Radio," IEICE Transactions on Communications, vol. E79-B, pp. 1262-1270, September 1996.

[26] D. Ramakrishna, N. Mandayam, and R. Yates, "Subspace Based Estimation of the Signal to Interference Ratio for CDMA Cellular Systems," IEEE Transactions on Vehicular Technology, vol. 49, September 2000. 


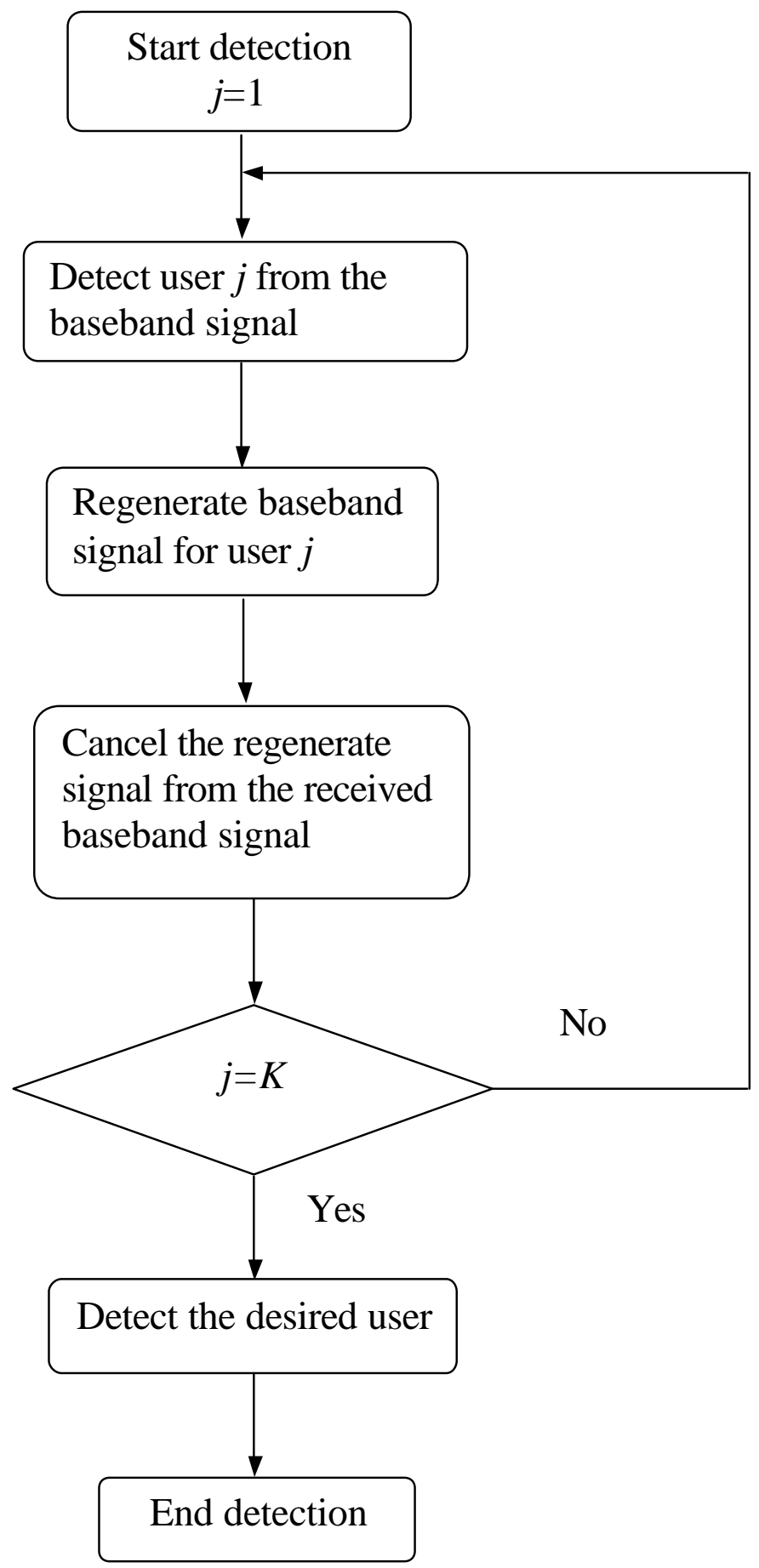

Figure 1: Flow chart illustrating the nonlinear centralized SIC scheme. 


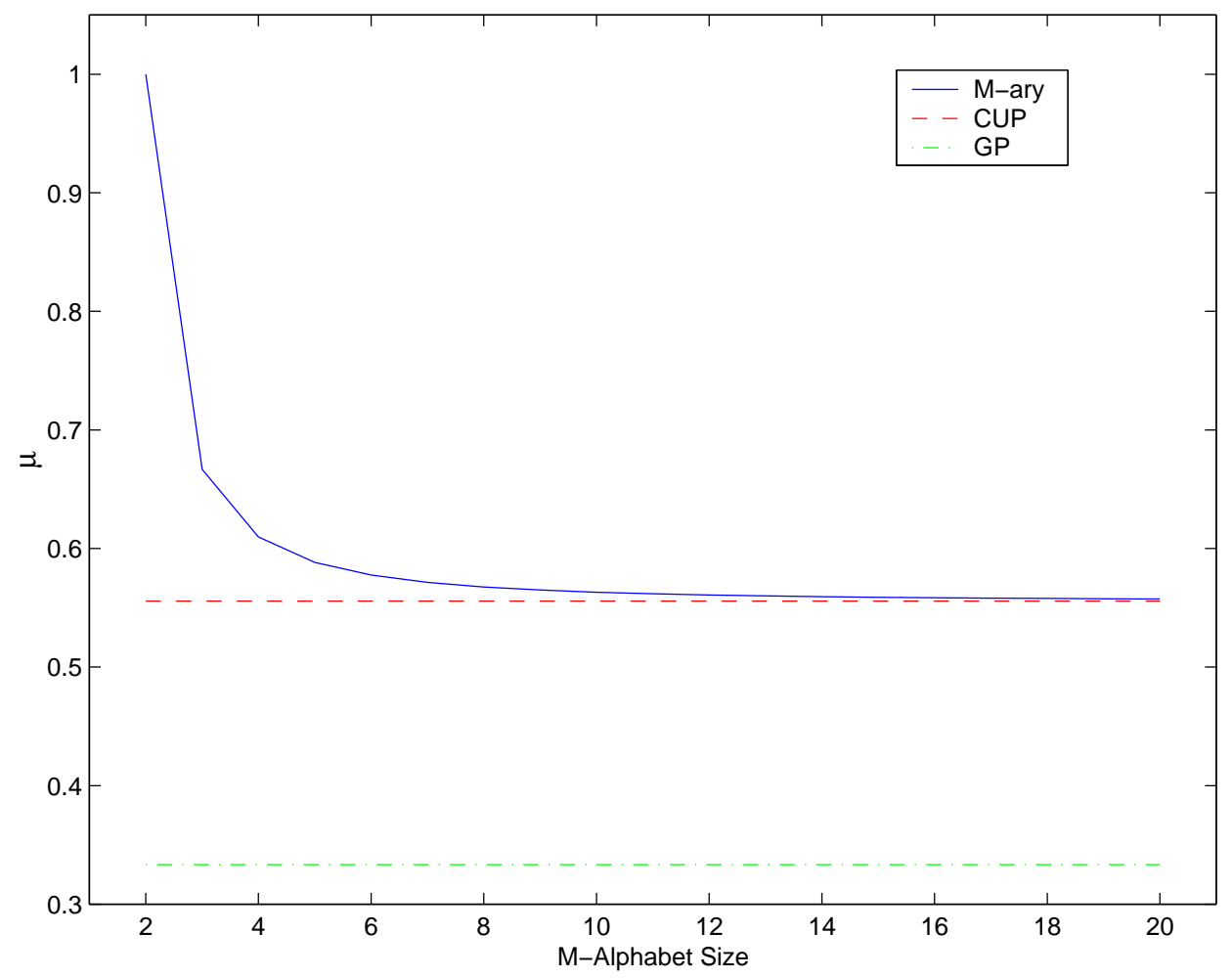

Figure 2: Parameter $\mu$ as a function of the alphabet size of a uniform, real-valued M-ary random process. 


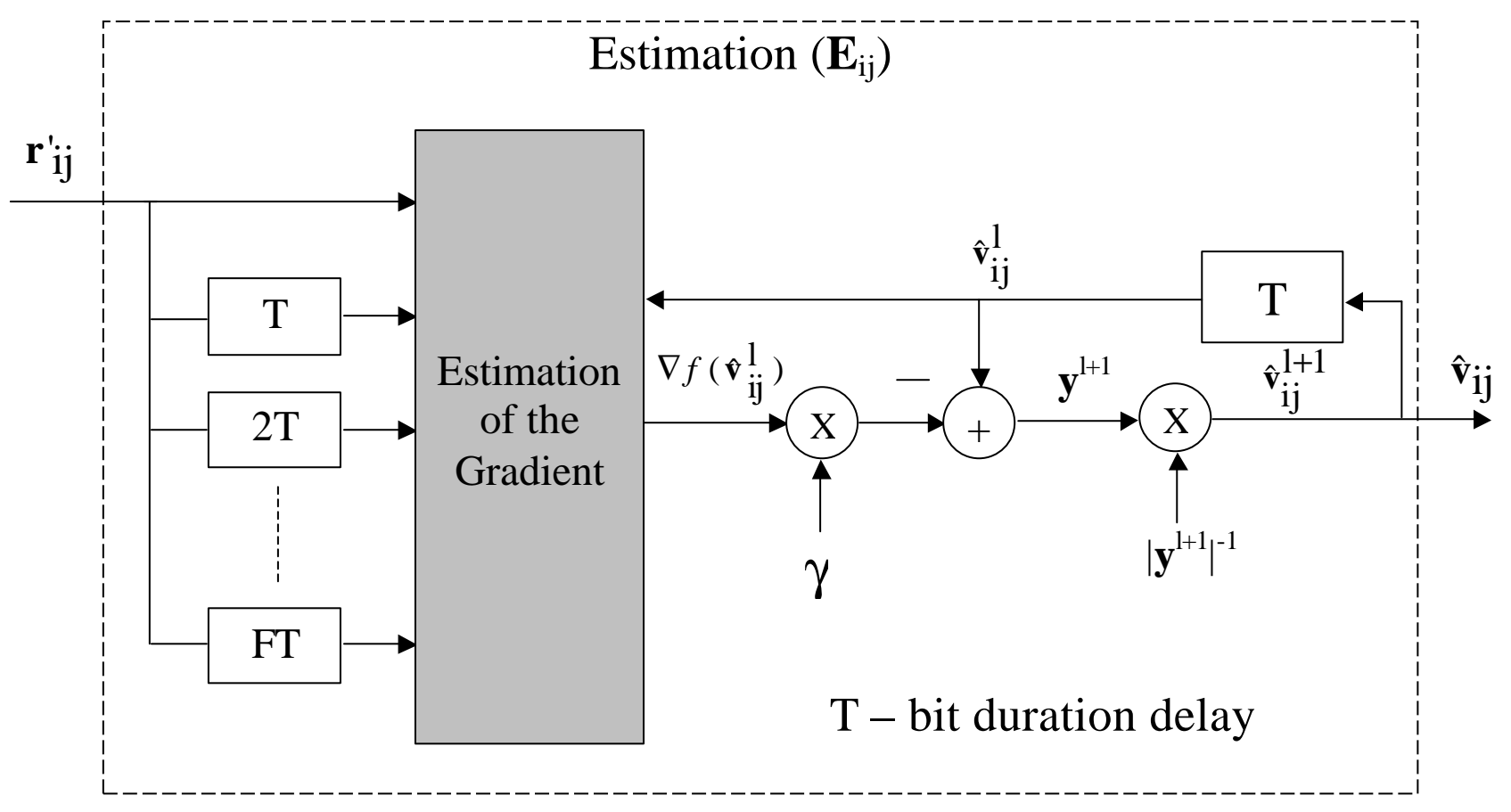

Figure 3: Block scheme: estimation of the vector $\mathbf{v}_{i j}$. 


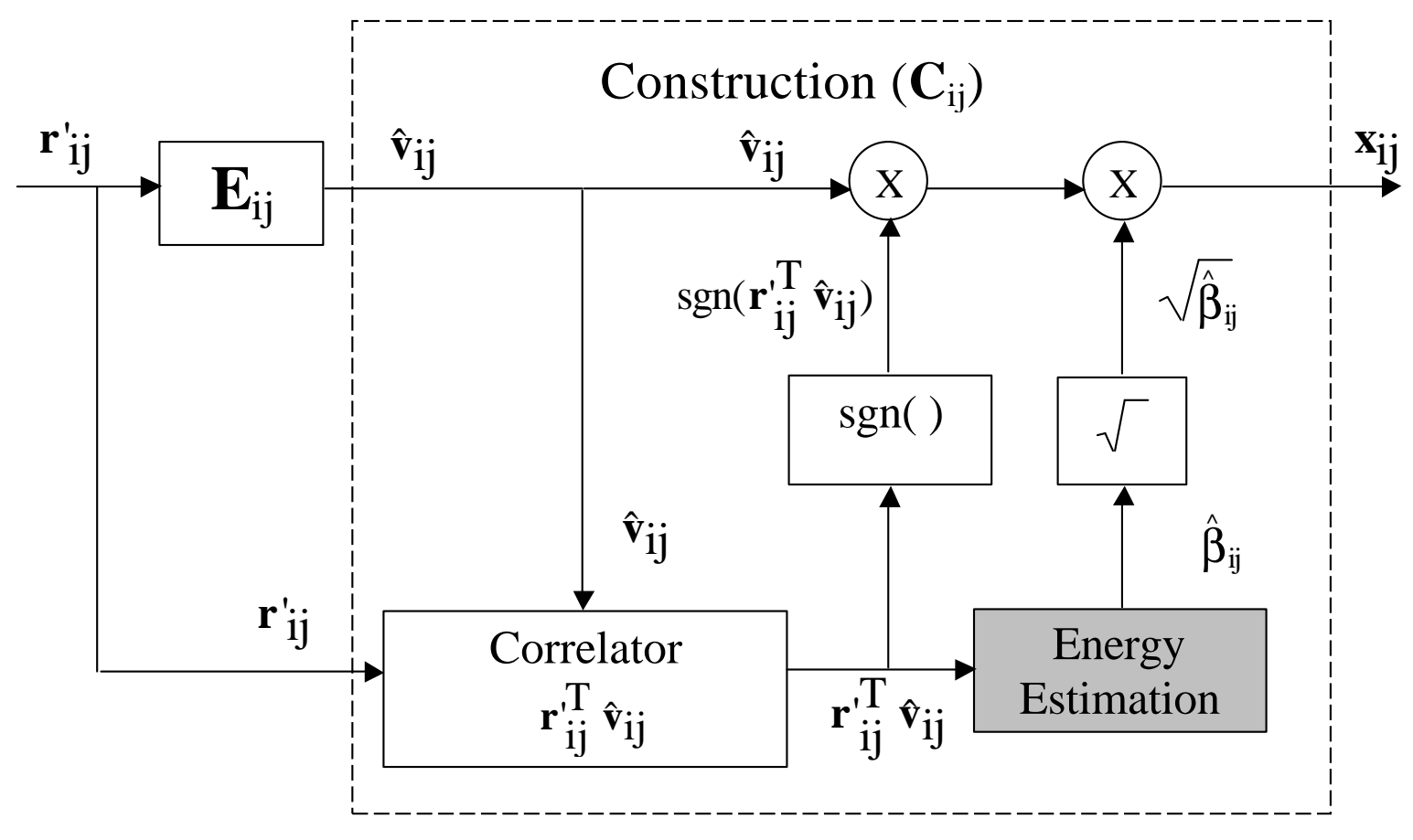

Figure 4: Block scheme: construction of the vector $\mathbf{x}_{i j}$. 


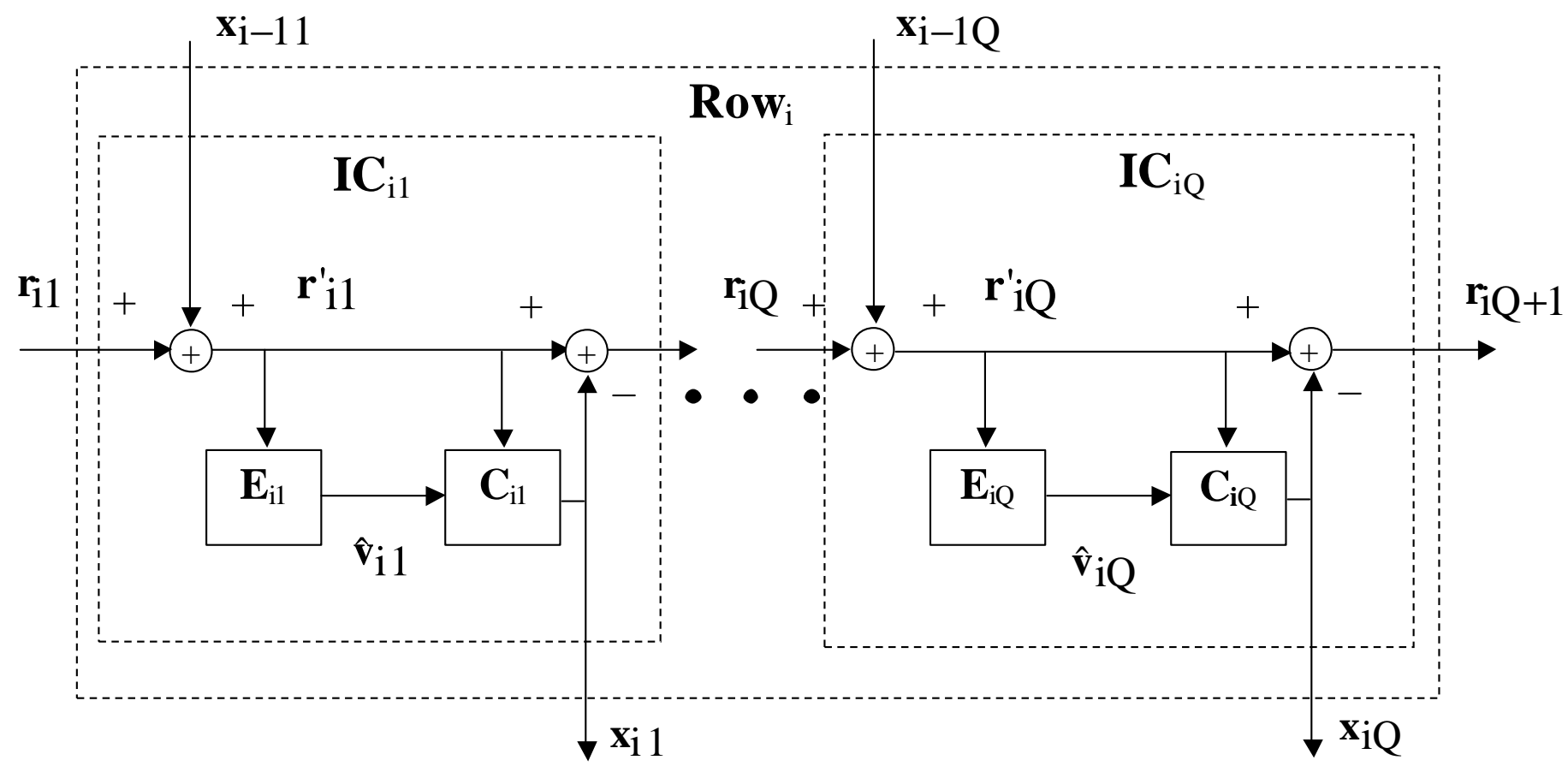

Figure 5: Horizontal topology of the MS-NL-BIC receiver. 


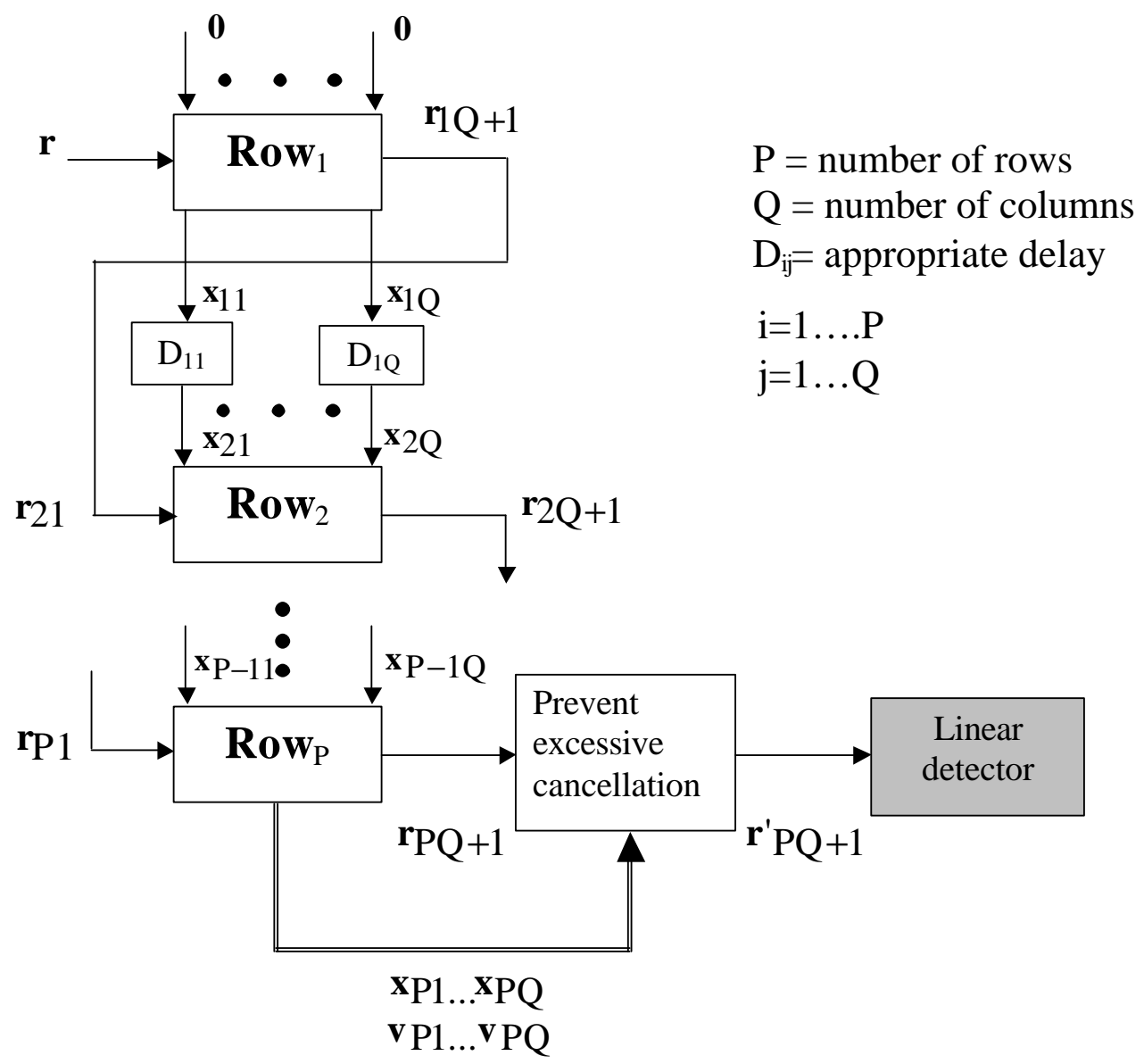

Figure 6: Vertical topology of the MS-NL-BIC receiver. 


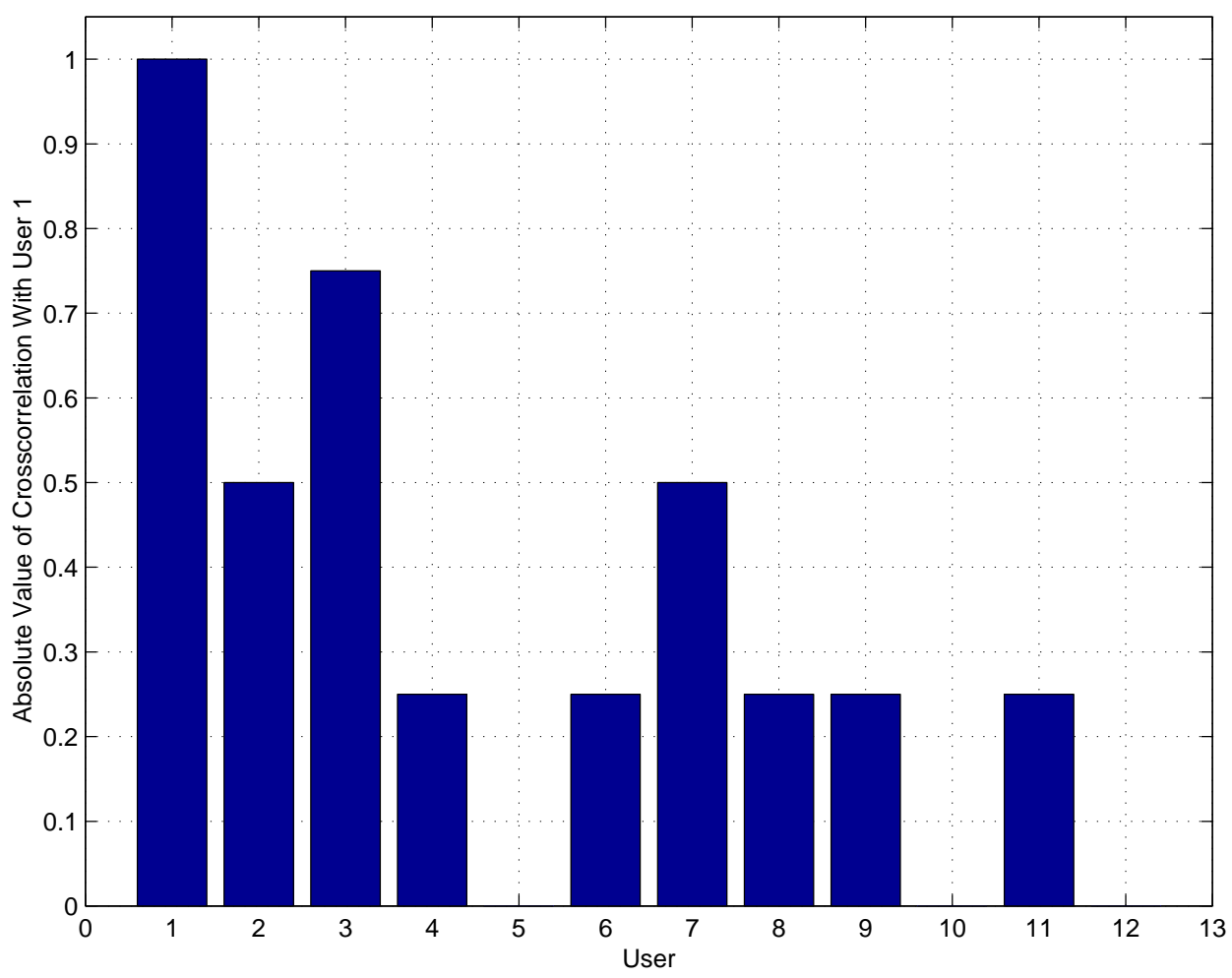

Figure 7: Absolute value of crosscorrelation between user 1 and user $\mathrm{i}(i=1, \cdots, 12)$. 


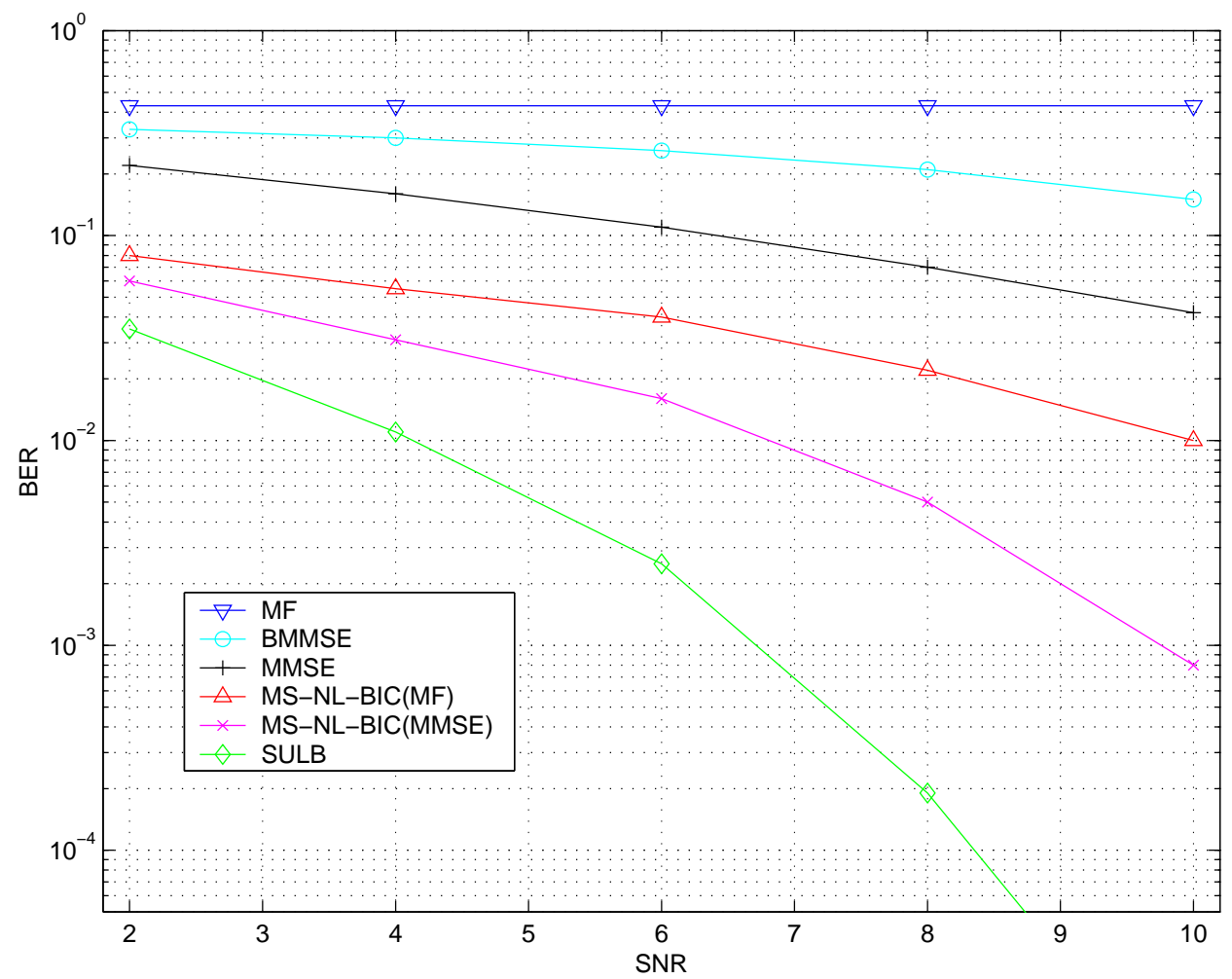

(a) BER vs. SNR, $L=8, M=8, A_{i}^{2} / A_{1}^{2}=25, i=2, \cdots, 8$.

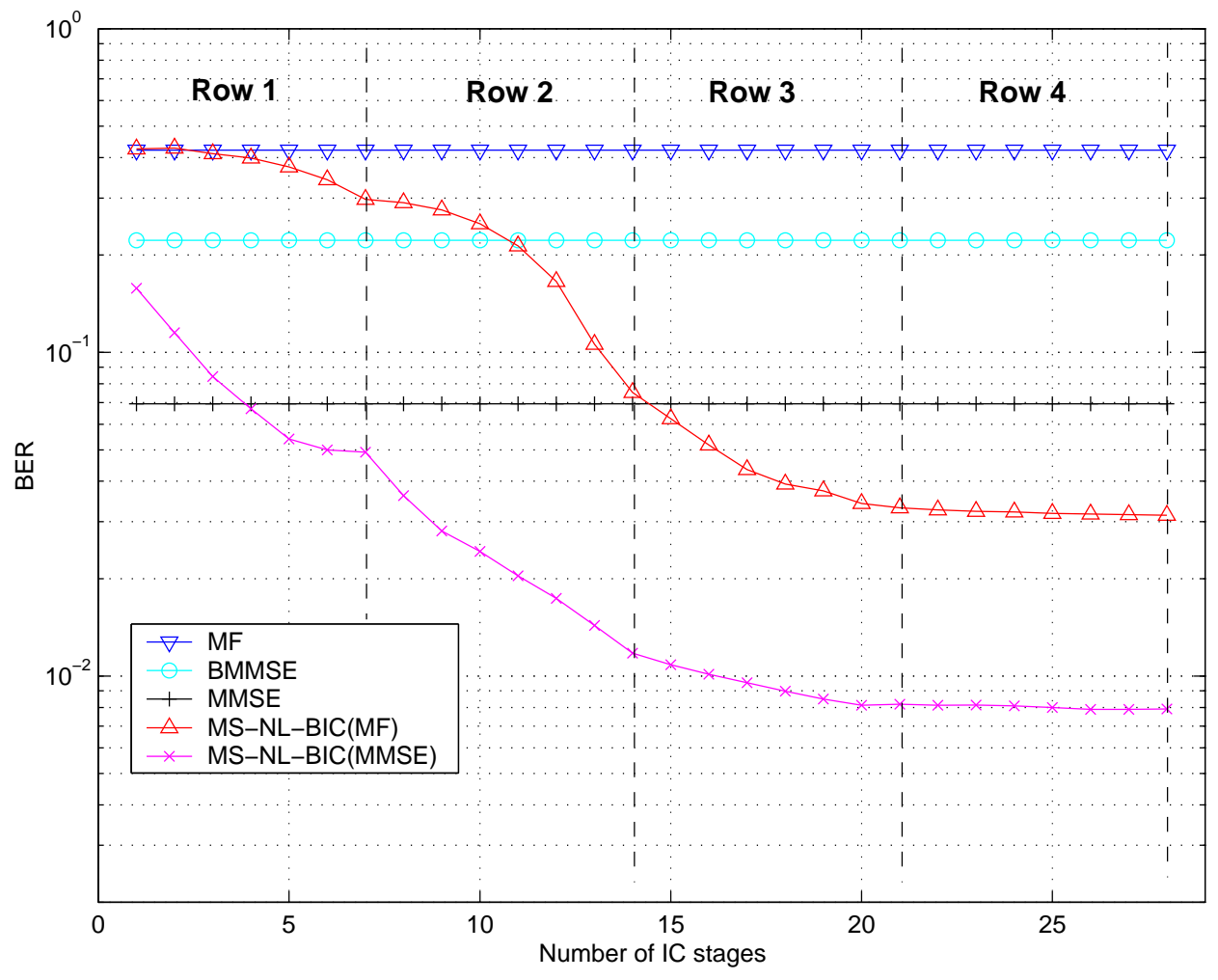

Figure 8: (b) BER vs. number of IC stages, $L=8, M=8, A_{i}^{2} / A_{1}^{2}=25, i=2, \cdots, 8, S N R=8 d B$. 


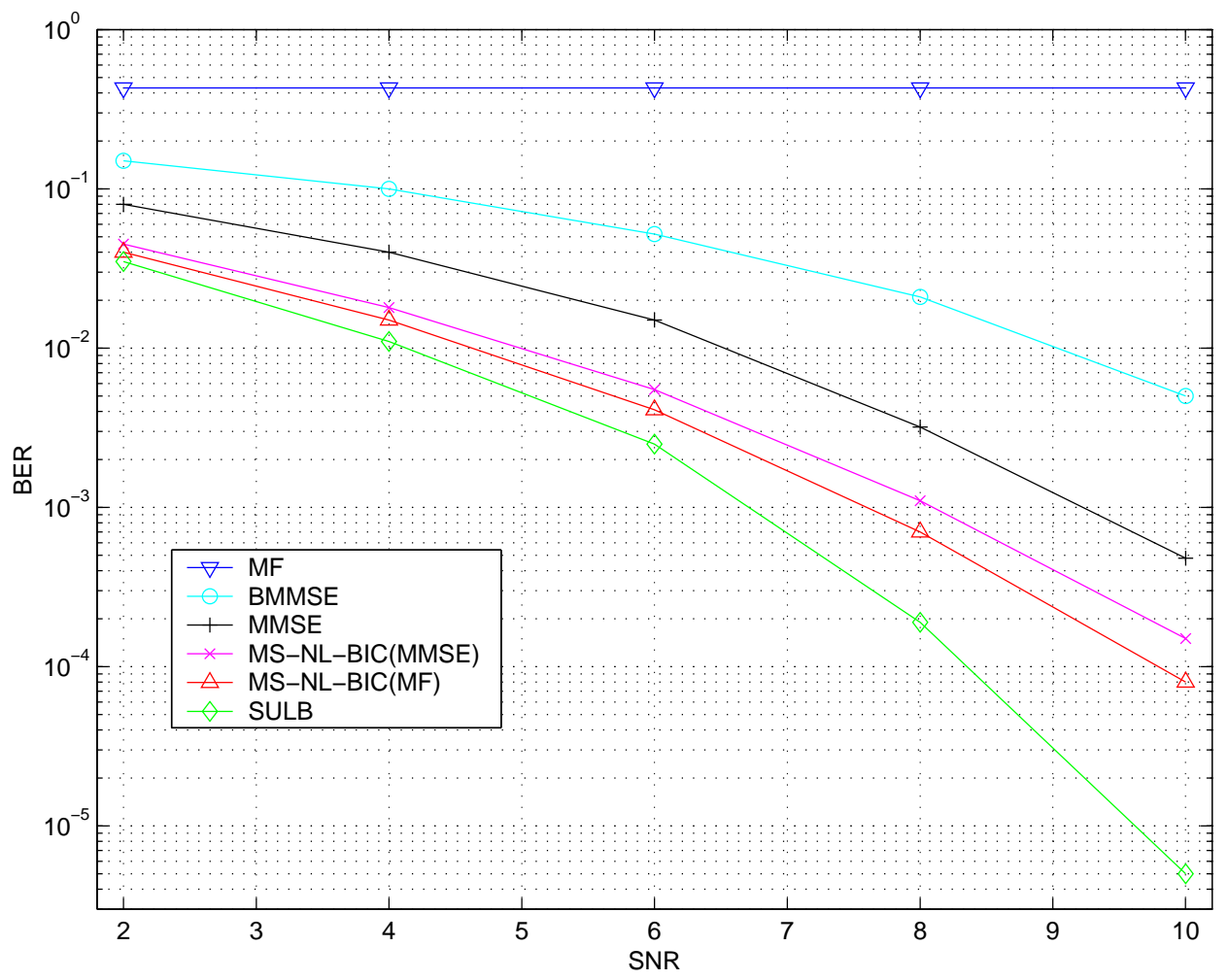

(a) BER vs. SNR, $L=4, M=8, A_{i}^{2} / A_{1}^{2}=25, i=2, \cdots, 4$.

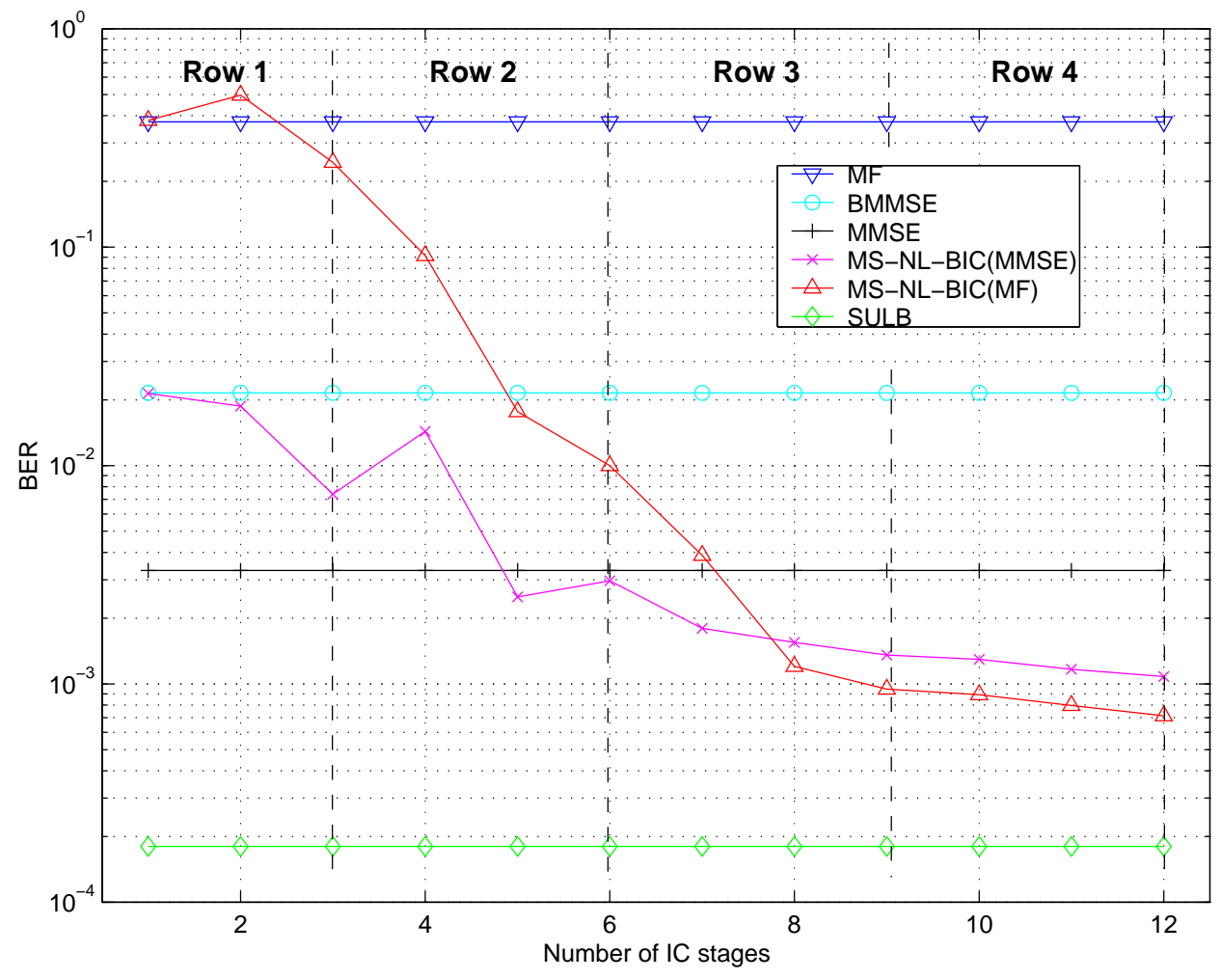

Figure 9: (b) BER vs. number of IC stages, $L=4, M=8, A_{i}^{2} / A_{1}^{2}=25, i=2, \cdots, 4, S N R=8 d B$. 


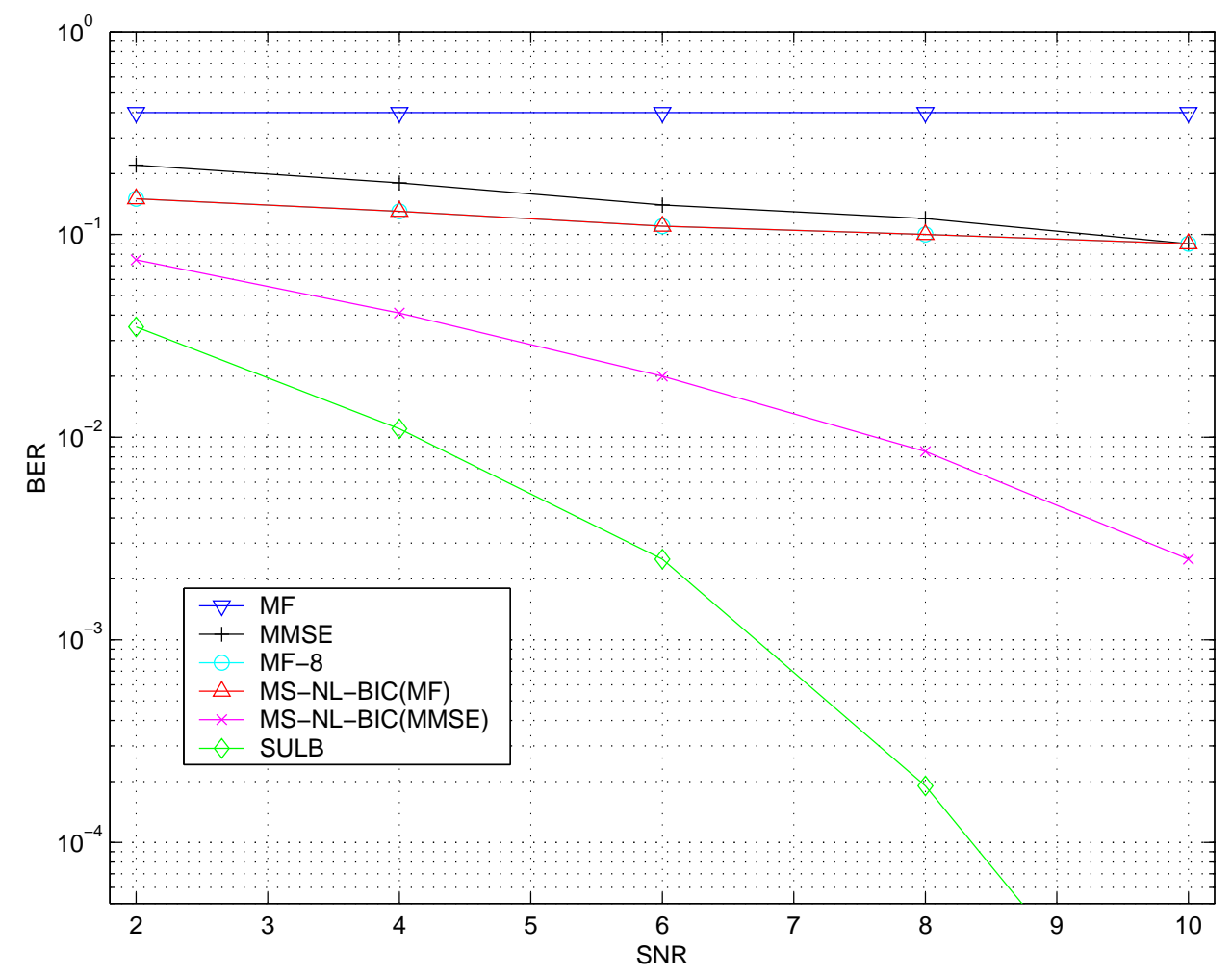

Figure 10: BER vs. SNR, $L=12, M=8, A_{i}^{2} / A_{1}^{2}=25, i=2, \cdots, 4$ and $A_{i}^{2} / A_{1}^{2}=1, i=5, \cdots, 12$. 


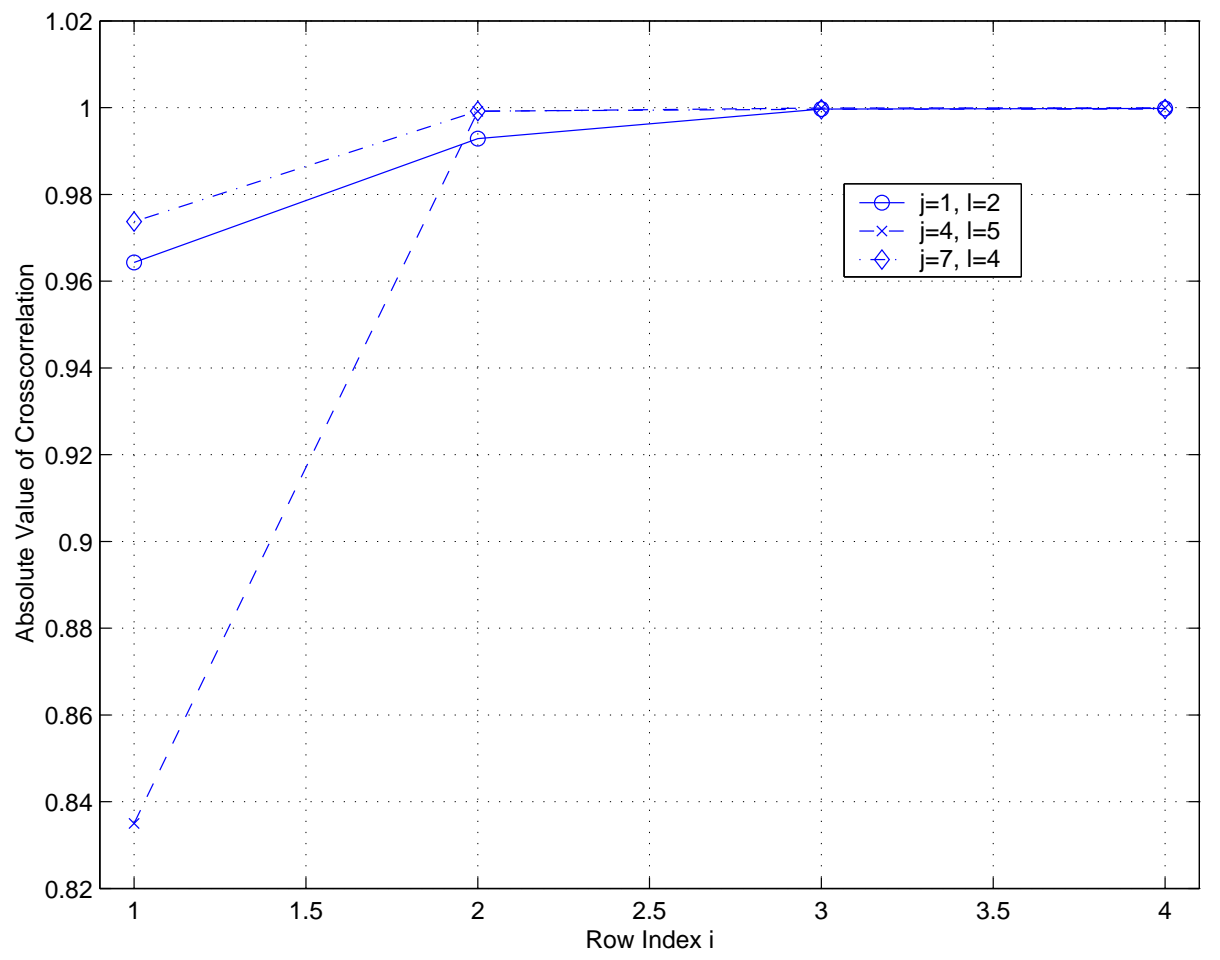

Figure 11: Absolute value of normalized crosscorrelation $\hat{\mathbf{v}}_{i j}^{\top} \mathbf{s}_{l}$, for columns $j=1,4,7$ and signature sequences $l=2,5,4$, respectively, $L=8, M=8, A_{i}^{2} / A_{1}^{2}=25, i=2, \cdots, 8$. 